\title{
Research Article \\ Fixed-Point Theory on a Frechet Topological Vector Space
}

\author{
Afif Ben Amar, ${ }^{1}$ Mohamed Amine Cherif, ${ }^{2}$ and Maher Mnif ${ }^{2}$ \\ ${ }^{1}$ Departement de Mathématiques, Faculté des Sciences de Gafsa, Université de Gafsa, \\ Cite Universitaire Zarrouk, Gafsa 2112, Tunisia \\ ${ }^{2}$ Departement de Mathématiques, Faculté des Sciences de Sfax, Université de Sfax, \\ Route de Soukra Km 3.5, B.P.1171, Sfax 3000, Tunisia
}

Correspondence should be addressed to Maher Mnif, maher.mnif@ipeis.rnu.tn

Received 6 December 2010; Revised 14 February 2011; Accepted 15 February 2011

Academic Editor: Genaro Lopez

Copyright (c) 2011 Afif Ben Amar et al. This is an open access article distributed under the Creative Commons Attribution License, which permits unrestricted use, distribution, and reproduction in any medium, provided the original work is properly cited.

\begin{abstract}
We establish some versions of fixed-point theorem in a Frechet topological vector space $E$. The main result is that every map $A=B C$ (where $B$ is a continuous map and $C$ is a continuous linear weakly compact operator) from a closed convex subset of a Frechet topological vector space having the Dunford-Pettis property into itself has fixed-point. Based on this result, we present two versions of the Krasnoselskii fixed-point theorem. Our first result extend the well-known Krasnoselskii's fixed-point theorem for $U$-contractions and weakly compact mappings, while the second one, by assuming that the family $\{T(\cdot, y): y \in C(M)$ where $M \subset E$ and $C: M \rightarrow E$ a compact operator $\}$ is nonlinear $\varphi$ equicontractive, we give a fixed-point theorem for the operator of the form $E x:=T(x, C(x))$.
\end{abstract}

\section{Introduction}

Fixed-point theorems are very important in mathematical analysis. They are an interesting way to show that something exists without setting it out, which sometimes is very hard, or even impossible to do. Several algebraic and topological settings in the theory and applications of nonlinear operator equations lead naturally to the investigation of fixedpoints of a sum of two nonlinear operators, or more generally, fixed-points of mappings on the cartesian product $E \times E$ into $E$, where $E$ is some appropriate space.

Fixed-point theorems in topology and nonlinear functional analysis are usually based on certain properties (such as complete continuity, monotonicity, contractiveness, etc.) that the operator, considered as a single entity must satisfy. We recall for instance the Banach fixed-point theorem, which asserts that a strict contraction on a complete metric space into 
itself has unique fixed-point, and the Schauder principle, which asserts that a continuous mapping $A$ on a closed convex set $M$ in Hausdorff locally convex topological vector space $E$ into $M$ such that $A(M)$ is contained in a compact set, has a fixed-point. In many problems of analysis, one encounters operators which may be split in the form $T=A+B$, where $A$ is a contraction in some sense, and $B$ is completely continuous, and $T$ itself has neither of these properties (see [1-3]). Thus neither the Schauder fixed-point theorem nor the Banach fixed-point theorem applies directly in this case, and it becomes desirable to develop fixedpoint theorems for such situations. An early theorem of this type was given by Krasnosel'skir [4]: "Let $E$ be a Banach space, $M$ be a bounded closed convex subset of $E$, and $A, B$ be operators on $M$ into $E$ such that $A x+B y \in M$ for every pair $x, y \in M$. If $A$ is a strict contraction and $B$ is continuous and compact, then the equation $A x+B x=x$ has a solution in M." This result has been extended to locally convex spaces in 1971 by Cain and Nashed [5]. There is also another theorem of this type which was given by Amar et al. [6] in 2005 and which extended the Schauder and Krasnoselskii fixed-point theorems in Dunford-Pettis spaces to weakly compact operators. Also in 2010, Amar and Mnif [7] established some new variants of Leray-Schauder type fixed-point theorems for weakly sequentially continuous operators.

In this paper, we give also a generalization of Krasnoselskii fixed-point theorems not in Dunford-Pettis Banach spaces but in Dunford-Pettis Frechet spaces. More precisely, let $E$ be a Frechet topological vector space having the property of Dunford-Pettis, $M$ a closed bounded convex subset of $E$, and $A=B C$ (where $B$ is a continuous map and $C$ is a linear weakly compact operator). If $A$ leaves $M$ invariant then $A$ has a fixed-point in $M$ (see Proposition 3.1). In addition, if $B$ is a $\varphi$-contraction map of $M$ into $E$, for each $x, y \in M$ with $B x+A y \notin M$, there is a $z \in(x, B x+A y) \cap M$ such that $B z+A y \in M$ and $(I-B)^{-1} A(M)$ is relatively weakly compact, then $A+B$ has a fixed-point in $M$ (see Proposition 3.3).

Based on our results and other theorems which was given by Sehgal and Singh in 1976 ([8]), we give also an extension of the Krasnoselskii fixed-point theorem: Let $E$ be a Frechet topological vector space having the property of Dunford-Pettis (DP), $M \subseteq E, C$ : $M \rightarrow C(M) \subseteq E$ a compact operator (An operator $C: M \rightarrow E$ is said to be compact if it is continuous and maps bounded sets into precompact.) and $T$ a map defined on the set $M \times C(M)$ and having range in $E$. By assuming that the family $\{T(\cdot, y): y \in C(M)\}$ is nonlinear $\varphi$ equicontractive we prove the existence of a point $x \in M$ such that

$$
x=T(x, C(x)) .
$$

Our paper is organized as follows. In Section 2, we give some important definitions and preliminaries which will be used in this paper. Among this preliminaries we cite definition of Dunford-Pettis space, the theorems of Schauder-Tychonoff, Krein-Smulian. The Section 3 is devoted to the generalization of the Krasnoselskii fixed-point theorem in Dunford-Pettis Frechet spaces where our proofs of our two results (Proposition 3.3 and Theorem 3.5) in this section are based on the theorem of Sehgal and Singh and the main result (Proposition 3.1).

\section{Preliminaries}

In this section, we give the following well-known definitions and results which will be used in this paper. 
Definition 2.1. Suppose that $E$ and $F$ are locally convex spaces. A continuous linear operator $A$ from $E$ into $F$ is said to be weakly compact if $A(B)$ is relatively weakly compact subset of $F$ whenever $B$ is a bounded subset of $E$.

Theorem 2.2 (Eberlein-S̃mulian, see [9]). Let E be a metrizable locally convex topological vector space, $\left(x_{n}\right)_{n}$ a weakly relatively compact sequence in $E$. Then from $\left(x_{n}\right)_{n}$ may be extracted a weakly convergent subsequence.

Definition 2.3. A subset $C$ in a vector space $E$ is called balanced if for all $x \in C, \lambda x \in C$ if $|\lambda| \leq 1$.

Definition 2.4 (see $[9,10]$ ). A locally convex topological vector space $E$ is said to have the Dunford-Pettis (DP) property if any continuous linear map of $E$ into a complete locally convex topological vector space $F$, which transforms bounded sets into weakly relatively compact sets, transforms each balanced and weakly compact subset of $E$ into a relatively compact subset of $F$.

Remark 2.5 (see [9]). If $E$ is complete, we replace in the precedent definition each balanced and weakly compact subset of $E$ by each weakly compact subset of $E$.

Theorem 2.6 (see [11]). Let E be a locally convex topological vector space and $M$ a convex subset of $E$. Then $M$ is closed if and only if it is weakly closed.

Theorem 2.7 (Krein-S̃mulian). Let E be a metrizable and complete locally convex topological vector space and $M \subset E$ weakly compact. Then the closed convex hull of $M$ is weakly compact.

Theorem 2.8 (Schauder-Tychonoff [12]). Let $M$ be a closed and convex subset of a locally convex topological vector space $E$ and $A: M \rightarrow M$ a continuous mapping such that the range $A(M)$ is contained in a compact set. Then $A$ has a fixed-point.

In the remainder of this section, $E$ denotes a Frechet topological vector space having the Dunford-Pettis (DP) property and $v$ is a neighborhood basis of the origin consisting of absolutely convex open subsets of $E$. Let for each $U \in \vartheta, p_{U}$ the Minkowski's functional of $\vartheta$.

Let $M$ be a nonempty subset of $E$. A mapping $A: M \rightarrow E$ is a $U$-contraction $(U \in \vartheta)$ if for each $\epsilon>0$ there is a $\delta>0$ such that if $x, y \in M$ and if

$$
x-y \in(\epsilon+\delta) U, \quad \text { then } A(x)-A(y) \in \epsilon U .
$$

If $A: M \rightarrow E$ is a $U$-contraction for each $U \in \vartheta$, then $A$ is a $\vartheta$-contraction.

Note that if $A$ is a $\vartheta$-contraction, then $A$ is continuous. (For a related definition of $\vartheta$-contraction, see Taylor [13].)

Lemma 2.9 (see [8]). Let $A: M \rightarrow E$ be a $v$-contraction, then $A$ is $\vartheta$-contractive, that is for each $U \in \vartheta, p_{U}(A(x)-A(y))<p_{U}(x-y)$ if $p_{U}(x-y) \neq 0$ and 0 , otherwise.

Theorem 2.10 (Theorem of Sehgal and Singh [8]). Let $M$ be a sequentially complete subset of $a$ complete separated locally convex topological vector space $F$ and $A: M \rightarrow F$ be a v-contraction. If $A$ 
satisfies the condition:

$$
\begin{aligned}
& \text { for each } x \in M \text { with } A(x) \notin M \text {, } \\
& \text { there is a } z \in(x, A(x)) \cap M \text { such that } A(z) \in M \text {. }
\end{aligned}
$$

Then A has a unique fixed-point in $M$.

Definition 2.11. Let $T: M \times E \rightarrow E$ be a map such that $M$ be a nonempty subset of $E$. The family $\{T(\cdot, y): y \in E\}$ is called $U$-equicontractive $(U \in \vartheta)$ if for each $\epsilon>0$ there is a $\delta>0$ such that if $\left(x_{1}, y\right),\left(x_{2}, y\right)$ in the domain of $T$ and if

$$
x_{1}-x_{2} \in(\epsilon+\delta) U, \quad \text { then } T\left(x_{1}, y\right)-T\left(x_{2}, y\right) \in \epsilon U
$$

If $\{T(\cdot, y): y\}$ is a $U$-equicontractive for each $U \in \vartheta$, then the family $\{T(\cdot, y): y\}$ is a $\vartheta$-equicontraction. Note that if the family $\{T(\cdot, y): y\}$ is a $\vartheta$-equicontraction, then the operator $x \rightarrow T(x, y)$ is a $\vartheta$-contraction for all $y$.

Definition 2.12. let $\varphi=\left\{p=p_{U}\right.$ for some $\left.U \in \vartheta\right\}, \mathbb{R}^{+}$the nonnegative reals and $\psi$ a family of mapping defined as $\psi=\left\{\Phi: \mathbb{R}^{+} \rightarrow \mathbb{R}^{+}\right.$such that $\Phi$ is continuous and $\Phi(t)<t$ if $\left.t>0\right\}$. A mapping $A: M \rightarrow E$ is a nonlinear $\varphi$ contraction (see [14]) if for each $p \in \varphi$, there is a $\Phi_{p} \in \psi$ such that $p(A(x)-A(y)) \leq \Phi_{p}(p(x-y))$ for all $x, y \in M$. If this inequality holds with $\Phi_{p}(t)=\alpha_{p} t$ such that $0<\alpha_{p}<1$, then $A$ is called $\varphi$-contraction (see [5]).

Since a nonlinear $\varphi$ contraction is a $\vartheta$-contraction, the following result immediately follows by Theorem 2.10 and provides an extension of a result in [5]:

Theorem 2.13 (see [8]). Let $M$ be a sequentially complete subset of a complete separated locally convex topological vector space $F$ and $A: M \rightarrow F$ be a nonlinear $\varphi$ contraction. If $A$ satisfies (2.2) then A has a unique fixed-point in $M$.

Definition 2.14. The family $\{T(\cdot, y): y \in E\}$ is called nonlinear $\varphi$ equicontractive if for each $p \in \varphi$, there is a $\Phi_{p} \in \psi$ such that if $\left(x_{1}, y\right),\left(x_{2}, y\right)$ in the domain of $T$, then

$$
p\left(T\left(x_{1}, y\right)-T\left(x_{2}, y\right)\right) \leq \Phi_{p}\left(p\left(x_{1}-x_{2}\right)\right) .
$$

Remark 2.15. Since any nonlinear $\varphi$ contraction is a $\vartheta$-contraction then any nonlinear $\varphi$ equicontraction is a $\vartheta$-equicontraction.

\section{Krasnoselskii's Type Theorems}

In this section, we will give some new fixed-point results for the sum of two operators where $E$ is a Frechet topological vector space having the Dunford-Pettis property. Firstly, we give the following proposition which is a generalization of Theorem 2.1 in [6]. 
Proposition 3.1. Let E be a Frechet topological vector space having the Dunford-Pettis property, $M$ a closed, bounded and convex subset of $E$ and $B, C$ two operators such that:

(i) $B: E \mapsto E$ a continuous map;

(ii) $C: E \mapsto E$ a linear weakly compact operator on $E$;

(iii) $B(C(M))$ is relatively weakly compact;

(iv) $A(M) \subset M$.

Then $A=B C$ has a fixed-point in $M$.

Proof. We denote by $N=\overline{\mathrm{co}}(A(M))$, the closed convex hull of $A(M)$. Firstly, we show that $N$ is a weakly compact subset of $E$. Indeed, we have $A(M) \subset \overline{B(C(M))}$. This implies that $A(M)$ is relatively weakly compact and therefore $\overline{A(M)}$ is weakly compact. We have

$$
A(M) \subset \overline{A(M)} \Longrightarrow N=\overline{\mathrm{co}}(A(M)) \subset \overline{\mathrm{co}}(\overline{A(M)})
$$

and since $\overline{A(M)}$ is weakly compact, then by Krein-S̃mulian's theorem $\overline{\mathrm{co}}(\overline{A(M)})$ is also weakly compact. Since $N$ is a closed convex subset of $E$, therefore it is weakly closed and this implies that $N$ is a weakly closed subset of a weakly compact. Consequently, $N$ is weakly compact.

Now, we show that $C(N)$ is relatively compact. We have $N$ is a weakly compact set in $E$ and $C$ is a weakly compact operator on $E$ and since $E$ is a Frechet topological vector space having the Dunford-Pettis property, then by Definition 2.4, we have $C(N)$ is a relatively compact set in $E$. Since $B$ is a continuous map, then $B C(N)$ is a relatively compact set in $E$.

Moreover, we have

$$
A(M) \subset M \quad \text { so } \overline{\mathrm{co}}(A(M)) \subset \overline{\mathrm{CO}}(M) \text {. }
$$

Therefore

$$
N=\overline{\mathrm{CO}}(A(M)) \subset M
$$

and this implies that

$$
A(N) \subset A(M) \subset \overline{\mathrm{co}}(A(M))=N,
$$

where $N$ is a closed convex and $A(N)=B C(N)$ is a relatively compact set. Since $C$ is a weakly compact oprator on $E$, then by Definition $2.1 C$ is continuous and so $A: N \rightarrow N$ is continuous. Finally, the use of Schauder-Tychonoff's fixed-point theorem shows that $A$ has at least one fixed-point in $N \subset M$.

Lemma 3.2. Let $E$ be a Frechet topological vector space, $M$ a sequentially complete subset of $E$ and $B$ : $M \mapsto E$ a nonlinear $\varphi$ contraction. Suppose that for $y \in E$ we have: for each $x \in M$ with $B x+y \notin M$, 
there is a $z \in(x, B x+y) \cap M$ such that $B z+y \in M$. Then, there exists a unique $u(y) \in M$ with $B(u(y))+y=u(y)$, that is $(I-B)^{-1} y=u(y) \in M$.

Proof. Consequence of Theorem 2.13 (see [8]).

The following proposition is a generalization of Theorem 2.2 in [6].

Proposition 3.3. Let E be a Frechet topological vector space having the Dunford-Pettis property, $M$ a closed, bounded and convex subset of $E$ and $A, B$ two operators such that:

(i) $A: E \mapsto E$ a linear weakly compact operator on $E$;

(ii) $B: M \mapsto E$ be a $\varphi$-contraction;

(iii) For each $x, y \in M$ with $B x+A y \notin M$, there is a $z \in(x, B x+A y) \cap M$ such that $B z+A y \in M$;

(iv) $(I-B)^{-1} A(M)$ is relatively weakly compact.

Then there exists $y$ in $M$ such that $A y+B y=y$

Proof. Firstly, we have $B$ is a $\varphi$-contraction then $B$ is a continuous function and for any $x, y \in$ $M$ we have

$$
p_{U}((I-B) x-(I-B) y) \geq p_{U}(x-y)-p_{U}(B x-B y) \geq\left(1-\alpha_{p}\right) p_{U}(x-y)
$$

with $\alpha_{p} \in(0,1)$ which gives the continuity of $(I-B)^{-1}$.

Now, by Lemma 3.2 equation $z=B z+A y$ has a unique solution $z \in M$ for all $y \in M$. It follows, that

$$
z=(I-B)^{-1} A y \in M,
$$

so

$$
(I-B)^{-1} A(M) \subset M .
$$

For conclusion, we have $(I-B)^{-1}$ is a continuous mapping, $A$ a linear weakly compact operator on $E$ and $(I-B)^{-1} A(M)$ is relatively weakly compact on $E$ where $(I-B)^{-1} A(M) \subset$ $M$. So, by Proposition 3.1, we prove that $(I-B)^{-1} A$ has a fixed-point in $M$ and this implies that, there exists $y \in M$ such that $A y+B y=y$.

We will now take $C: M \rightarrow C(M) \subseteq E$ a compact operator and $T$ a map defined on the set $M \times C(M)$ and having range in $E$. We are interested to the existence of a point $x \in M \subset E$ such that

$$
x=T(x, C(x)) .
$$


Proposition 3.4. Let $E$ be a Frechet topological vector space, $M$ a bounded sequentially complete subset of $E$ and

$$
T: M \times E \longrightarrow E
$$

a map such that the family $\{T(\cdot, y): y \in E\}$ is nonlinear $\varphi$ equicontractive, for all $x \in M, y \rightarrow$ $T(x, y)$ is continuous and which satisfies the condition: for each $(x, y) \in M \times E$ with $T(x, y) \notin M$, there is a

$$
z \in(x, T(x, y)) \cap M \text { such that } T(z, y) \in M
$$

Then there exists a continuous map $F_{T}: E \rightarrow M$ such that

$$
T\left(F_{T}(y), y\right)=F_{T}(y)
$$

Proof. We start from an arbitrary point $y \in E$. Since the family $\{T(\cdot, y): y \in E\}$ is a nonlinear $\varphi$ equicontractive then the operator

$$
x \longrightarrow T(x, y): M \longrightarrow E \text { is a nonlinear } \varphi \text { contraction }
$$

which satisfy for each $x \in M$ with $T(x, y) \notin M$, there is a

$$
z \in(x, T(x, y)) \cap M \quad \text { such that } T(z, y) \in M
$$

Then by Theorem 2.13, there is a unique point $x=F_{T}(y) \in M$ that satisfies the operator equation:

$$
T\left(F_{T}(y), y\right)=F_{T}(y)
$$

We will show that the mapping $y \mapsto F_{T}(y): E \rightarrow M$ is continuous. To do this we let $\left(y_{n}\right)$ be a sequence in $E$, with $\lim y_{n}=y_{0} \in E$. We suppose that $F_{T}\left(y_{n}\right)$ does not converge to $F_{T}\left(y_{0}\right)$. Then there exist $p \in \varphi$, an $\epsilon>0$ and $\rho(n)$ such that

$$
p\left(F_{T}\left(y_{\rho(n)}\right), F_{T}\left(y_{0}\right)\right)>\epsilon \quad \forall n \in \mathbb{N} .
$$


Since $\left\{p\left(F_{T}\left(y_{\rho(n)}\right), F_{T}\left(y_{0}\right)\right)>\epsilon, n \in \mathbb{N}\right\}$ is a bounded real subsequence, it has a subsequence $\left\{p\left(F_{T}\left(y_{\left.\rho_{\left(\rho_{1}(n)\right)}\right)}\right), F_{T}\left(y_{0}\right)\right), n \in \mathbb{N}\right\} \rightarrow r \geq 0$. However, we have

$$
\begin{aligned}
p\left(F_{T}\left(y_{\rho\left(\rho_{1}(n)\right)}\right)-F_{T}\left(y_{0}\right)\right)= & p\left(T\left(F_{T}\left(y_{\rho\left(\rho_{1}(n)\right)}\right), y_{\rho\left(\rho_{1}(n)\right)}\right)-T\left(F_{T}\left(y_{0}\right), y_{0}\right)\right) \\
\leq & p\left(T\left(F_{T}\left(y_{\rho\left(\rho_{1}(n)\right)}\right), y_{\left.\rho\left(\rho_{1}(n)\right)\right)}\right)-T\left(F_{T}\left(y_{0}\right), y_{\rho((n))}\right)\right) \\
& +p\left(T\left(F_{T}\left(y_{0}\right), y_{\rho\left(\rho_{1}(n)\right)}\right)-T\left(F_{T}\left(y_{0}\right), y_{0}\right)\right) \\
\leq & \Phi_{p}\left(p\left(F_{T}\left(y_{\rho\left(\rho_{1}(n)\right)}\right)-F_{T}\left(y_{0}\right)\right)\right) \\
& +p\left(T\left(F_{T}\left(y_{0}\right), y_{\rho\left(\rho_{1}(n)\right)}\right)-T\left(F_{T}\left(y_{0}\right), y_{0}\right)\right)
\end{aligned}
$$

which implies that $r=0$. This contradicts (3.14) and consequently $F_{T}$ is continuous.

In what follows, we give also another result of Krasnoselskii type.

Theorem 3.5. Let $M$ be a closed, bounded and convex subset of a Frechet topological vector space having the Dunford-Pettis property $E$ and $C: M \rightarrow E$ a linear weakly compact operator such that the image of $C(M)$ by any continuous mapping is contained in a weakly compact subset of $E$. Let

$$
T: M \times C(M) \longrightarrow E
$$

be a map such that the family $\{T(\cdot, y): y \in C(M)\}$ is nonlinear $\varphi$ equicontractive, for all $x \in M$, $y \mapsto T(x, y)$ is continuous on $C(M)$ and which satisfies that for each $(x, y) \in M \times C(M)$ with $T(x, y) \notin M$, there is a

$$
z \in(x, T(x, y)) \cap M \text { such that } T(z, y) \in M
$$

Then $(\mathrm{H})$ admits a solution in $M$.

Proof. We start from an arbitrary point $y \in C(M)$. By Proposition 3.4 we prove that there exists a unique point $x=F_{T}(y) \in M$ that satisfies the operator equation

$$
T\left(F_{T}(y), y\right)=F_{T}(y)
$$

where the mapping $y \mapsto F_{T}(y): C(M) \rightarrow M$ is continuous. Then the operator $F_{T} C$ maps the set $M$ into itself. We have by hypothesis that $F_{T}(C(M))$ is contained in a weakly compact subset of $E$. Therefore, by Proposition 3.1, we prove the existence of a point $\bar{x} \in M$ such that $F_{T}(C(\bar{x}))=\bar{x}$. This means that

$$
T(\bar{x}, C(\bar{x}))=T\left(F_{T}(C(\bar{x})), C(\bar{x})\right)=F_{T}(C(\bar{x}))=\bar{x}
$$




\section{References}

[1] T. A. Burton, "A fixed-point theorem of Krasnoselskii," Applied Mathematics Letters, vol. 11, no. 1, pp. 85-88, 1998

[2] T. A. Burton and C. Kirk, "A fixed point theorem of Krasnoselskii-Schaefer type," Mathematische Nachrichten, vol. 189, pp. 23-31, 1998.

[3] B. C. Dhage, "Local fixed point theory for the sum of two operators in Banach spaces," International Journal on Fixed Point Theory, Computation and Applications, vol. 4, no. 1, pp. 49-60, 2003.

[4] M. A. Krasnosel'skiř, "Two remarks on the method of successive approximations," Uspekhi Matematicheskikh Nauk, vol. 10, no. 1(63), pp. 123-127, 1955.

[5] G. L. Cain, Jr. and M. Z. Nashed, "Fixed points and stability for a sum of two operators in locally convex spaces," Pacific Journal of Mathematics, vol. 39, pp. 581-592, 1971.

[6] A. B. Amar, A. Jeribi, and M. Mnif, "On a generalization of the Schauder and Krasnosel'skii fixed points theorems on Dunford-Pettis spaces and applications," Mathematical Methods in the Applied Sciences, vol. 28, no. 14, pp. 1737-1756, 2005.

[7] A. B. Amar and M. Mnif, "Leray-Schauder alternatives for weakly sequentially continuous mappings and application to transport equation," Mathematical Methods in the Applied Sciences, vol. 33, no. 1, pp. 80-90, 2010.

[8] V. M. Sehgal and S. P. Singh, "On a fixed point theorem of Krasnoselskii for locally convex spaces," Pacific Journal of Mathematics, vol. 62, no. 2, pp. 561-567, 1976.

[9] R. E. Edwards, Functional Analysis. Theory and Applications, Holt, Rinehart and Winston, New York, NY, USA, 1965.

[10] J.-L. Clerc and Y. C. De Verdière, "Compacité faible dans les espaces localement convexes; applications aux espaces $\mathrm{C}(\mathrm{K})$ et $\mathrm{L}^{1}(\mu)$, , Séminaire Choquet. Initiation à l'Analys, vol. 7, no. 2, 1967-1968.

[11] C. D. Aliprantis and K. C. Border, Infinite Dimensional Analysis: A Hitchhiker's Guide, Cataloging-inPublication Data. Library of Congress Control Number: 2006921177.

[12] A. Tychonoff, "Ein Fixpunktsatz," Mathematische Annalen, vol. 111, no. 1, pp. 767-776, 1935.

[13] W. W. Taylor, "Fixed-point theorems for nonexpansive mappings in linear topological spaces," Journal of Mathematical Analysis and Applications, vol. 40, pp. 164-173, 1972.

[14] D. W. Boyd and J. S. W. Wong, "On nonlinear contractions," Proceedings of the American Mathematical Society, vol. 20, pp. 458-464, 1969. 


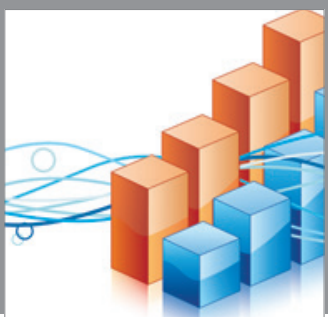

Advances in

Operations Research

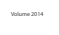

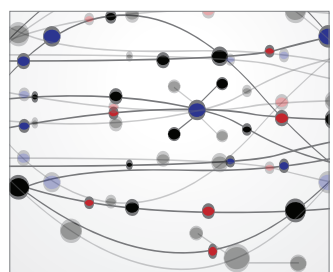

\section{The Scientific} World Journal
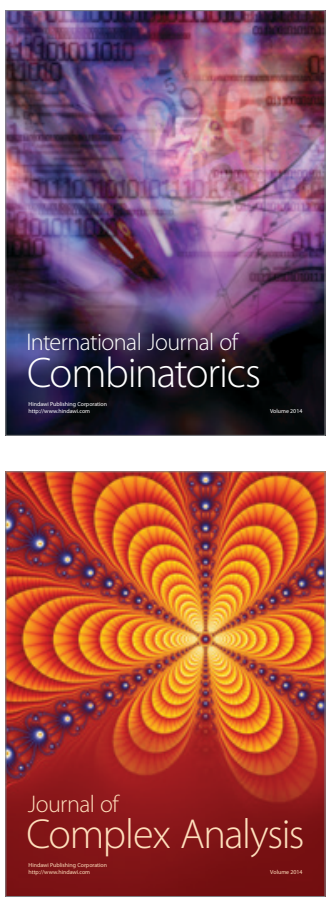

International Journal of

Mathematics and

Mathematical

Sciences
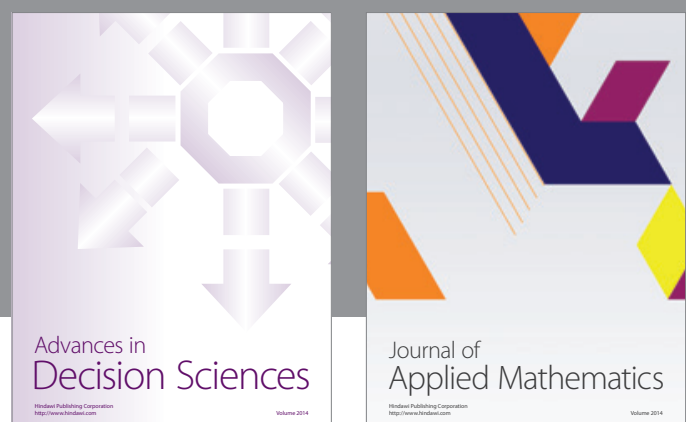

Journal of

Applied Mathematics
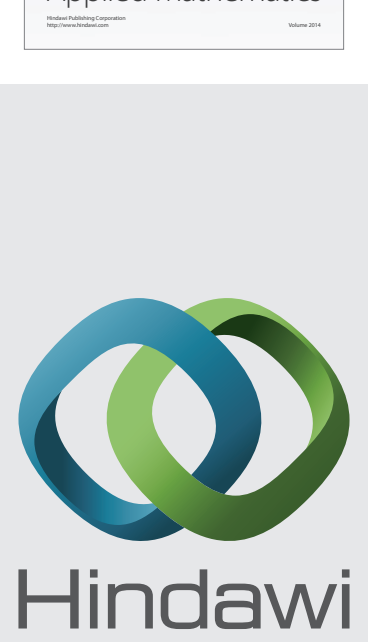

Submit your manuscripts at http://www.hindawi.com
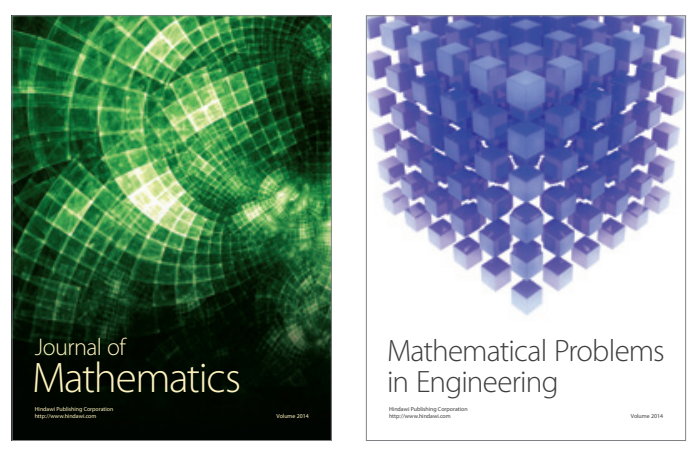

Mathematical Problems in Engineering
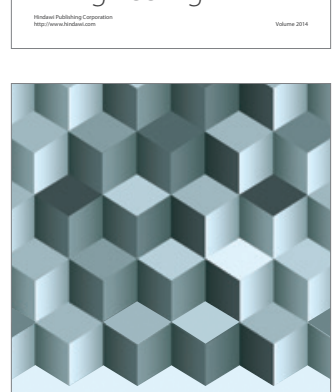

Journal of

Function Spaces
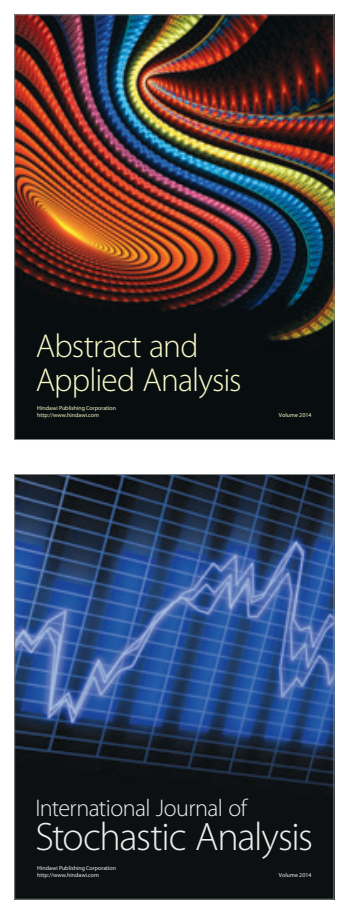

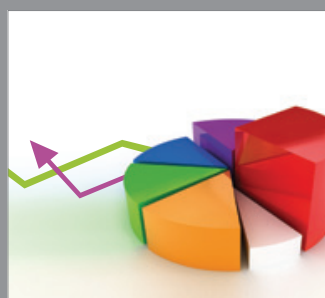

ournal of

Probability and Statistics

Promensencen
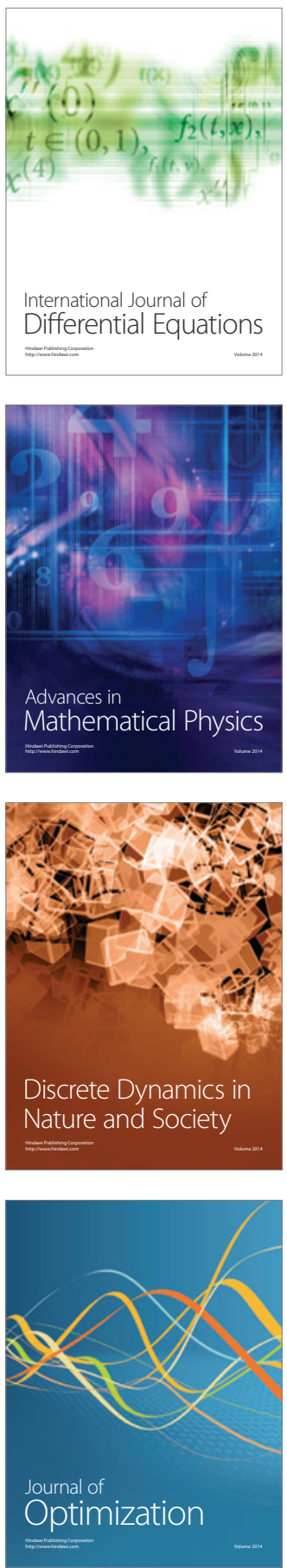